\title{
Images - Well-differentiated papillary mesothelioma of the tunica vaginalis
}

Ailsa May Li Gan, BSc ${ }^{1}$; Paul Plantinga, MD²; Nahid Punjani, MD, MPH${ }^{3}$; Andrew Hussey, MD FRCSC ${ }^{4}$; Nicholas Power, MD, FRCSC ${ }^{1,3}$

${ }^{1}$ Schulich School of Medicine and Dentistry, Western University, London, ON; ${ }^{2}$ Department of Pathology and Laboratory Medicine, London Health Sciences Centre, London, ON; ${ }^{3}$ Division of Urology, Department of Surgery, Western University, London, ON; ${ }^{4}$ Uroloist, Stratford, ON; Canada

Cite as: Can Urol Assoc J 2018 May 14; Epub ahead of print. http://dx.doi.org/10.5489/cuaj.4940

Published online May 14, 2018

$* * *$

\section{Introduction}

Well-differentiated papillary mesothelioma (WDPM) is a rare mesothelial tumour occurring in the tunica vaginalis of the testicle. The pathological classification of paratesticular mesothelial tumours is controversial and continues to evolve in the literature. Diagnostic criteria of one subtype, WDPM, are histological and include the following: 1) papillary or tubular-papillary architecture; 2) bland nuclear cytology; 3) low mitotic activity; 4) lined by a single row of cuboidal cells; and 5) absence of stromal invasion., ${ }^{1,2}$ This report presents issues of diagnosis of this rare pathology with imaging from an example case.

\section{Issues of diagnosis}

Rarity

Bárbera and Rubino first demonstrated the possibility of mesotheliomas to occur within the tunica vaginalis in 1957, describing a benign papillary tumour displaying histological features resembling mesothelial lesions occurring in other serous membranes. ${ }^{3}$ Since then, it has become evident that the incidence of this exact localization is rare and reported to comprise less than $1 \%$ of mesotheliomas. ${ }^{4}$ Given the uncommonness of this disease process, and thus the further rarity of mesothelioma subtypes, current understanding of this disease's clinical and pathological features is limited.

Non-specific presentation and risk factors

Typical presentation is with a painless and firm testicular mass often with an associated hydrocele. ${ }^{2,5-8}$ Asbestos exposure is considered as a possible risk factor due to its longstanding known association with pleural mesothelioma. ${ }^{9}$ However, a similar correlation has not been found in development of WDPM of the testes in the previously reported cases. ${ }^{2,8,10}$ Pre-operative diagnosis of mesothelial lesions of the tunica vaginalis is difficult due to broad differential 
diagnoses, and therefore, radical orchiectomy is the current recommended initial management, relying on surgical pathology for definitive diagnosis. Figure 1 shows a photomicrograph of a wedge biopsy sent intraoperatively for cryosection. The biopsy was reported as a neoplastic epithelial lesion and mesothelioma could not be excluded.

\section{Histological uncertainty}

The diagnosis of malignant mesothelioma of the tunica vaginalis is confirmed by histological criteria that fall into three subtypes (epithelial, biphasic, and sarcomatous) and are described by the prominent architecture patterns (e.g. tubulopapillary, adenomatoid, solid epithelioid or pleomorphic). ${ }^{4}$ Mesothelial origin can be confirmed using immunohistochemical stains. Figure 2 shows lesional cells displaying positive calretinin, WT-1, HBME-1, and D2-40; focally positive MOC-31 and BER-EP4; and negative B72.3 staining.

The classification uncertainty arises when microscopic examination reveals a specimen with complex morphology that lacks overt signs of malignancy, thus failing to meet the criteria for malignant mesothelioma. ${ }^{11}$ Figure 3 displays microscopic findings on surgical pathology from our example case; the specimen appeared to be composed of nodules with exophytic papillary architecture, lined predominantly by a single layer of bland, cuboidal, eosinophilic cells. The cells displayed minimal atypia, and no mitotic figures were identified. There was no invasion of the stroma or tunica.

The diagnostic uncertainty of histopathology is discussed in the literature and demonstrated through discrepancy between studies of previously reported cases. Brimo et al. reviewed a series of eight cases of para-testicular mesotheliomas with a range of histologic features that do not meet the classification of malignant mesothelioma, recommending that the diagnosis of WDPM be reserved for cases with the previously listed criteria (see Introduction) and that lack any atypical or complex pathology. ${ }^{12}$ Several authors ${ }^{6,713}$ suggest cases that do not meet the criteria be termed "mesothelioma of uncertain malignant potential," a term that has yet to be officially adopted. In 2016, two case reports with literature reviews were published concerning WDPM of the tunica vaginalis; Tan et al. ${ }^{2}$ described only seven "true" cases of WDPM of 24 total reported cases they reviewed, while Parcesepe et al. ${ }^{10}$ reported a total of seventeen WDPM cases. Interestingly, only four cases were common between the two., 3,7,13,14

\section{Prognostic implication}

In the literature, three categories of mesothelial tumours have been proposed: well-differentiated papillary mesothelioma, mesothelioma of uncertain malignant potential, and malignant mesothelioma. ${ }^{12}$ WDPM is considered a benign neoplasm with an indolent course ${ }^{2}$, while malignant mesothelioma can exhibit aggressive behaviour, having been reported to present local recurrence in up to $60 \%$ of cases within the first 2 years after initial treatment, and up to $90 \%$ by 5 years. ${ }^{10}$ It should be noted that follow-up time from previously reported cases of WDPM has been variable, ranging from 2 months to 10 years, with no reported recurrence following surgical 
management. ${ }^{2,11}$ Careful identification, documentation, and follow-up of this pathology are imperative to confirm that this is truly a benign lesion.

\section{Conclusion}

Due to the continued uncertainty surrounding diagnostic classification and the stark differences in prognostic implication between diagnoses, it is imperative to continue to report and document pathological and immunohistochemical findings in order to develop a greater understanding of these lesions. Long-term follow up studies would be beneficial for future cases to observe possible correlations in presentation, pathological characteristics, and immunohistochemical markers with malignancy potential to better guide treatment and follow-up. 


\section{References}

1. Chen X, Sheng W, Wang J. Well-differentiated papillary mesothelioma: A clinicopathological and immunohistochemical study of 18 cases with additional observation. Histopathology 2013;62:805-13.

2. Tan WK, Tan M-Y, Tan WS, et al. Well-Differentiated papillary mesothelioma of the tunica vaginalis: Case report and systematic review of literature. Clin Genitourin Cancer 2016;14:e435-9.

3. Bárbera V, Rubino M. Papillary mesothelioma of the tunica vaginalis. Cancer 1957;10:183-9.

4. Attanoos RL, Gibbs AR. Primary malignant gonadal mesotheliomas and asbestos. Histopathology 2000;37:150-9.

5. Tolhurst SR, Lotan T, Rapp DE, et al. Well-differentiated papillary mesothelioma occurring in the tunica vaginalis of the testis with contralateral atypical mesothelial hyperplasia. Urol Oncol Semin Orig Investig 2006;24:36-9.

6. Butnor KJ, Sporn TA, Hammar SP, et al. Well-differentiated papillary mesothelioma. Am J Surg Pathol 2001;25:1304-9.

7. Fukunaga M. Well-differentiated papillary mesothelioma of the tunica vaginalis: A case report with aspirate cytologic, immunohistochemical, and ultrastructural studies. Pathol Res Pract 2010;206:105-9.

8. Erdogan S, Acikalin A, Zeren H, et al. Well-differentiated papillary mesothelioma of the tunica vaginalis: A case study and review of the literature. Korean J Pathol 2014;48:2258.

9. Wagner J, Sleggs C, Marchand P. Diffuse pleural mesothelioma and asbestos exposure in the North Western Cape Province. Br J Ind Med 1960;17:260-71.

10. Parcesepe P, Sina S, Zanella C, et al. Case report of a well-differentiated papillary mesothelioma of the tunica vaginalis in an undescended testis with review of literature. Int J Surg Pathol 2016;24:443-7.

11. Trpkov K, Barr R, Kulaga A, et al. Mesothelioma of tunica vaginalis of "uncertain malignant potential" - an evolving concept: case report and review of the literature. Diagn Pathol 2011;6:78.

12. Brimo F, Illei PB, Epstein JI. Mesothelioma of the tunica vaginalis: a series of eight cases with uncertain malignant potential. Mod Pathol 2010;23:1165-72.

13. Fujii $Y$, Masuda M, Hirokawa $M$, et al. A case of benign mesothelioma of the tunica vaginalis testis. Acta Urol Jpn 1993;39:89-92.

14. Chollet Y, Hauser P, Da Silva T, et al. Well-differentiated papillary mesothelioma of the tunica vaginalis testis: Imaging on Tc-99m heat-denatured red blood cell scintigraphy. Clin Nucl Med 2008;33:282-4. 


\section{Figures and Tables}

Fig. 1. Photomicrograph of lesion submitted for intraoperative consultation (cryosection), showing papillary excrescences on the surface of the tunica vaginalis (hematoxylin and eosin, 10x magnification).

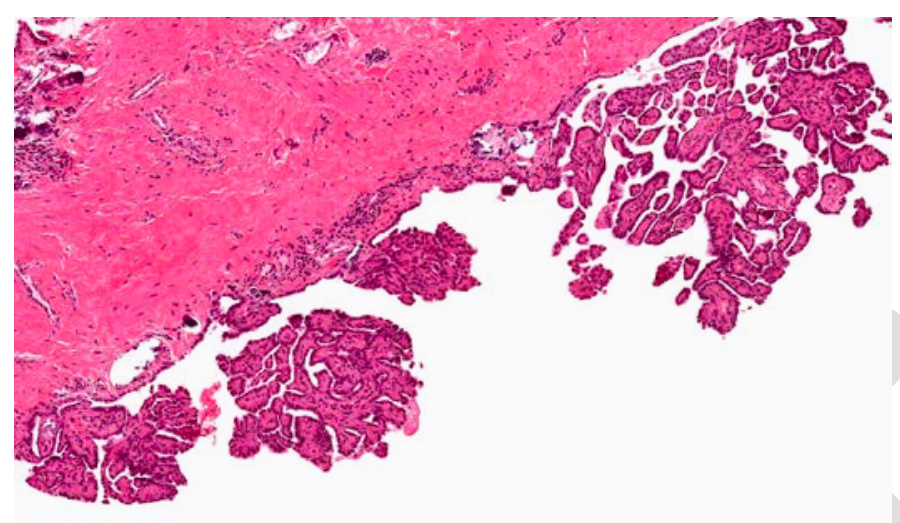


Fig. 2. Photomicrograph demonstrating mesothelial origin, with lesional cells positive by immunohistochemistry for (a) calretinin; (b) D2-40; and (c) WT-1 (4x magnification).

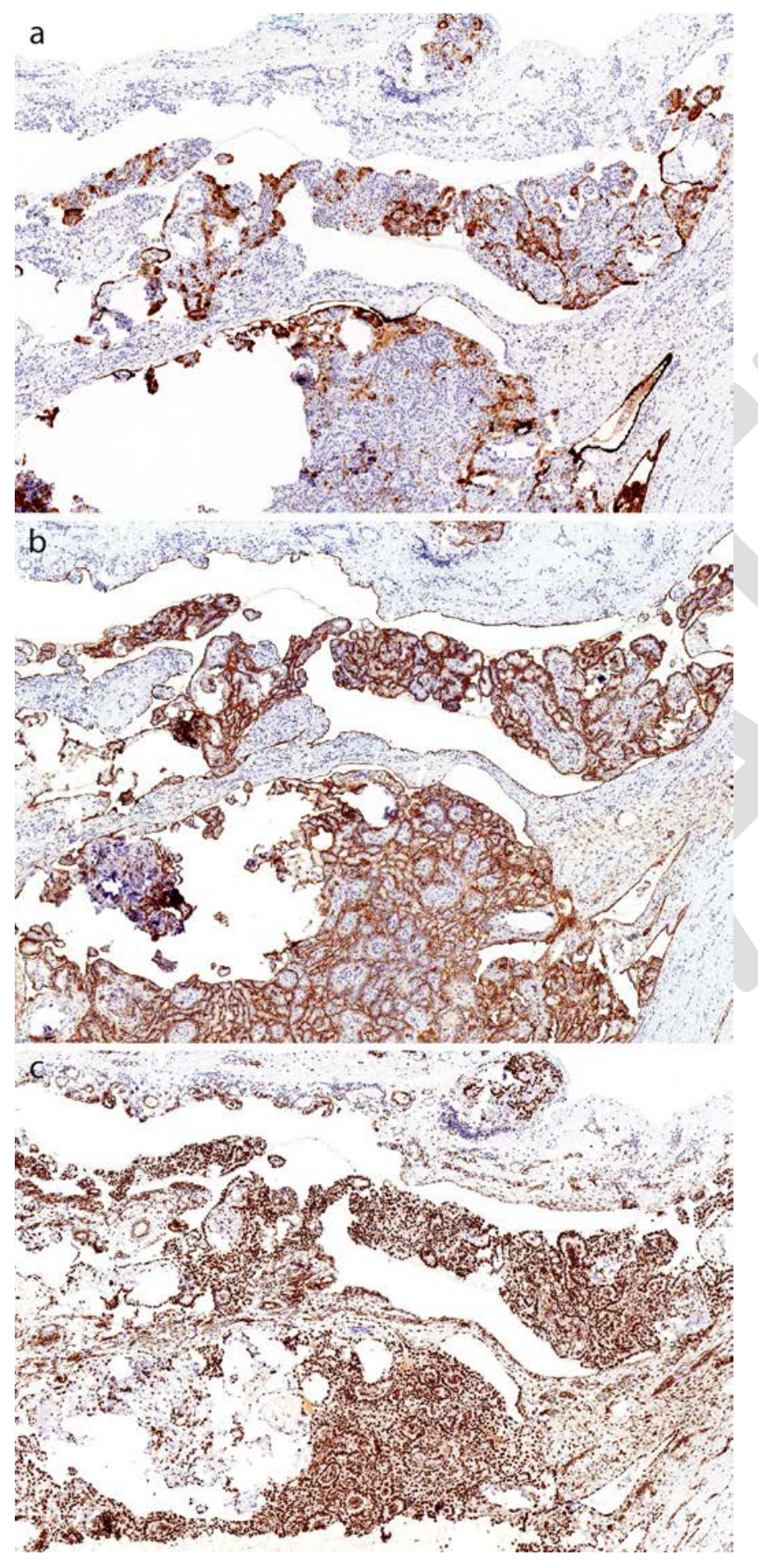


Fig. 3. Photomicrograph of papillary lesion involving the tunica vaginalis, with no invasion, lined by a single layer of cuboidal epithelium; with adjacent testis in (a) (hematoxylin and eosin, at (a) 4x; (b) 10x; and (c) 20x magnification).
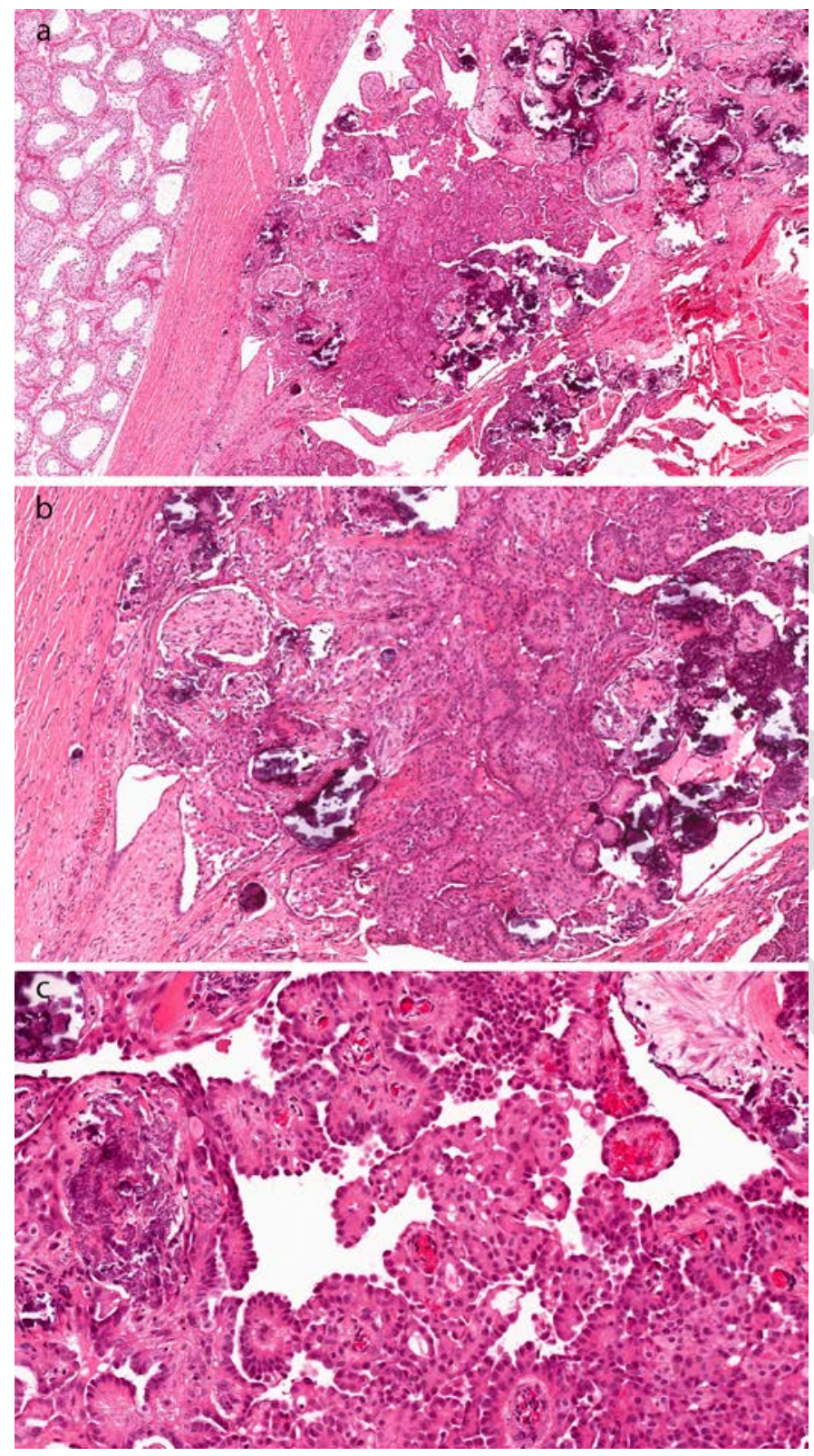